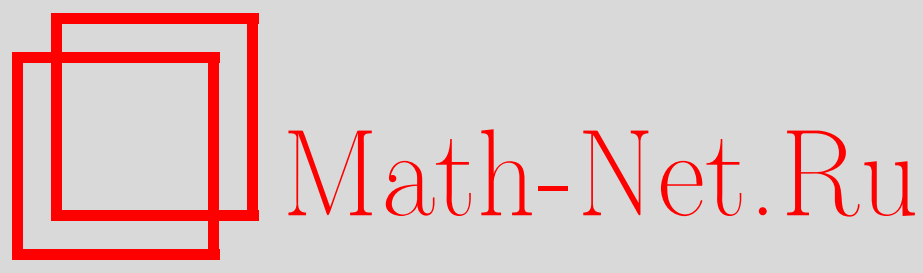

В. Л. Верещагин, Дискретные цепочки Тоды и метод Лапласа, ТМФ, 2009, том 160, номер 3, 434-443

DOI: https://doi.org/10.4213/tmf6408

Использование Общероссийского математического портала Math-Net.Ru подразумевает, что вы прочитали и согласны с пользовательским соглашением http://www . mathnet.ru/rus/agreement

Параметры загрузки:

IP: 3.82 .47 .9

26 апреля 2023 г., 12:00:19

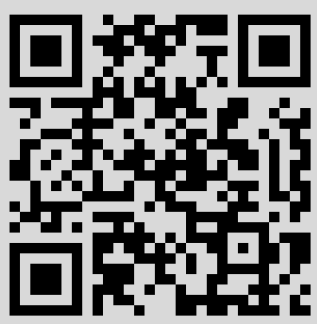




\section{ДИСКРЕТНЫЕ ЦЕПОЧКИ ТОДЫ И МЕТОД ЛАПЛАСА}

Каскадный метод Лапласа применен к системам дискретных уравнений вида $u_{i+1, j+1}=f\left(u_{i+1, j}, u_{i, j+1}, u_{i j}, u_{i, j-1}\right)$, где $u_{i j}, i, j \in \mathbb{Z},-$ элементы последовательности неизвестных векторов. Введено понятие обобщенного инварианта Лапласа и связанного с ним свойства “лиувиллевости" системы. Доказан ряд утверждений о корректности определения обобщенного инварианта и его применимости для поиска решений и интегралов системы. Приведены примеры дискретных систем типа Лиувилля.

Ключевые слова: нелинейные дискретные уравнения, метод Лапласа, интегрируемость по Дарбу.

\section{1. ВВЕДЕНИЕ}

Каскадный метод, предложенный Лапласом в 1773 г., позволяет находить общее решение специальных линейных гиперболических уравнений второго порядка с переменными коэффициентами вида

$$
u_{x y}+a(x, y) u_{x}+b(x, y) u_{y}+c(x, y) u=0, \quad u=u(x, y)
$$

Суть метода может быть кратко изложена следующим образом. Величины $h=$ $a_{x}+a b-c$ и $k=b_{y}+a b-c$ называются инвариантами Лапласа и служат исходным пунктом для каскадного метода. Если хотя бы один из инвариантов $h$ или $k$ тождественно равен нулю, то исходное уравнение (1.1) легко интегрируется в квадратурах. В случае неравенства нулю обоих инвариантов замена

$$
u=\frac{1}{h}\left(\frac{\partial}{\partial x}+b\right) u_{1}
$$

переводит исходное уравнение (1.1) в уравнение того же типа на функцию $u_{1}$ :

$$
\frac{\partial^{2} u_{1}}{\partial x \partial y}+a_{1}(x, y) \frac{\partial u_{1}}{\partial x}+b_{1}(x, y) \frac{\partial u_{1}}{\partial y}+c_{1}(x, y) u_{1}=0
$$

${ }^{*}$ Институт математики с ВЦ УНЦ РАН, Уфа, Россия. E-mail: v_vereschagin@mail.ru 
где $a_{1}=a-(\ln h)_{y}, b_{1}=b, c_{1}=a_{1} b_{1}+b_{y}-h$. Аналогично для уравнения (1.2) также имеется инвариант Лапласа $h_{1}=2 h-k-(\ln h)_{x y}$. Переход от уравнения $(1.1)$ к $(1.2)$ называется преобразованием Лапласа. Подобным образом при $k \neq 0$ заменой

$$
u=\frac{1}{k}\left(\frac{\partial}{\partial y}+a\right) u_{-1}
$$

из (1.1) выводим линейное уравнение

$$
\frac{\partial^{2} u_{-1}}{\partial x \partial y}+a_{-1}(x, y) \frac{\partial u_{-1}}{\partial x}+b_{-1}(x, y) \frac{\partial u_{-1}}{\partial y}+c_{-1}(x, y) u_{-1}=0
$$

где $a_{-1}=a, b_{-1}=b-(\ln k)_{x}, c_{-1}=a_{-1} b_{-1}+a_{x}-k$, для которого соответствующий инвариант Лапласа определяется по формуле $k_{-1}=2 k-h-(\ln k)_{x y}$. Имеется связь между инвариантами:

$$
h_{1}=2 h-k-(\ln h)_{x y}, \quad h_{-1}=k, \quad k_{1}=h, \quad k_{-1}=2 k-h-(\ln k)_{x y} .
$$

Если хотя бы один из инвариантов $h_{1}$ и $k_{-1}$ равен нулю, получаем уравнение, интегрируемое в квадратурах и, следовательно, квадратурную формулу для решения исходного уравнения (1.1). Если же $h_{1}$ и $k_{-1}$ не равны нулю, то снова применяем к $u_{1}$ и $u_{-1}$ преобразование Лапласа, что дает цепочку уравнений и соответствующих инвариантов Лапласа

$$
\ldots, h_{-2}, h_{-1}, h=h_{0}, h_{1}, h_{2}, \ldots
$$

при условии $h_{-1}=k$ и $k_{n}=h_{n-1}$. Вся цепочка инвариантов может быть вычислена с помощью рекуррентной формулы

$$
\left(\ln h_{j}\right)_{x y}=h_{j+1}-2 h_{j}+h_{j-1}, \quad j \in \mathbb{Z},
$$

представляющей собой одну из форм записи двумеризованной цепочки Тоды.

Известно, что и в теории нелинейных уравнений второго порядка вида

$$
u_{x y}=f\left(x, y, u, u_{x}, u_{y}\right)
$$

инварианты Лапласа соответствующего линеаризованного уравнения

$$
\left(\frac{\partial^{2}}{\partial x \partial y}-f_{u_{x}} \frac{\partial}{\partial x}-f_{u_{y}} \frac{\partial}{\partial y}-f_{u}\right) v=0
$$

также являются важным инструментом [1]-[4]. В частности, доказано, что уравнение обладает нетривиальными интегралами по каждому из характеристических направлений ( $x$ - и $y$-интегралами, т.е. величинами $I\left(x, y, u, u_{x}, u_{y}\right), J\left(x, y, u, u_{x}, u_{y}\right)$ такими, что $I_{x}=J_{y}=0$ вдоль траекторий уравнения (1.6)) тогда и только тогда, когда ряд инвариантов Лапласа уравнения обрывается нулями с обеих сторон. Это обстоятельство позволило связать метод Лапласа с теорией интегрируемости по Дарбу. 
ОПРЕДЕЛЕНИЕ 1. Уравнение (1.6) называется интегрируемъмм по Дарбу, если у него существуют нетривиальные $x$-интегралы и $y$-интегралы.

Наиболее известными примерами уравнений, интегрируемых по Дарбу, являются волновое уравнение $u_{x y}=0$ (интегралы $I=u_{y}, J=u_{x}$ ) и уравнение Лиувилля $u_{x y}=e^{u}$ (интегралы $\left.I=u_{y y}-u_{y}^{2} / 2, J=u_{x x}-u_{x}^{2} / 2\right)$. Отметим, что уравнение Лиувилля получается из цепочки Тоды (1.5) путем простого замыкания цепочки инвариантов: $h_{j}=0$ при $j \neq 0, h_{0}=-e^{u} / 2$. В работе [5] было показано, что аналогичные построения можно провести и в дискретной ситуации, в частности для дискретных линейных уравнений вида

$$
u_{i+1, j+1}=f\left(u_{i+1, j}, u_{i, j+1}, u_{i j}\right) .
$$

В работе [5] также был описан аналог метода Лапласа, построены дискретные инварианты и показано, что эти инварианты удовлетворяют уравнению

$$
\left(h_{i, j+1}^{k-1}+1\right)\left(h_{i+1, j}^{k+1}+1\right)=\left(\frac{1}{h_{i, j+1}^{k}}+1\right)\left(\frac{1}{h_{i+1, j}^{k}}+1\right) h_{i j}^{k} h_{i+1, j+1}^{k},
$$

представляющему собой дискретную двумеризованную цепочку Тоды. Простейшим нетривиальным замыканием уравнения (1.9) и, следовательно, дискретным уравнением Лиувилля ${ }^{1)}$ является уравнение

$$
u_{i+1, j+1}\left(1+\frac{1}{u_{i+1, j}}\right)\left(1+\frac{1}{u_{i, j+1}}\right) u_{i j}=1 .
$$

В последние десятилетия проводились активные исследования интегрируемых по Дарбу нелинейных гиперболических систем уравнений вида

$$
u_{x y}^{i}=F^{i}\left(x, y, u, u_{x}, u_{y}\right), \quad i=1,2, \ldots, n,
$$

в частности дискретной цепочки Тоды (см. [6]-[12]). Были предложены определения инвариантов Лапласа и систем, интегрируемых по Дарбу, и показано, что основные идейные положения метода Лапласа остаются в силе и для систем типа (1.11).

В работе [13] был построен аналог метода Лапласа для систем нелинейных дискретных уравнений вида

$$
u_{i+1, j+1}^{l}=F^{l}\left(u_{i+1, j}, u_{i, j+1}, u_{i j}\right), \quad l=1,2, \ldots, n .
$$

Достигнутые результаты ясно показывают почти полное совпадение основных идейных положений метода Лапласа в применении к непрерывным уравнениям второго порядка вида (1.1), (1.6), (1.11) и к дискретным цепочкам (1.8), (1.12), связывающим значения неизвестных последовательностей в четырех соседних точках решетки $(i, j),(i+1, j),(i, j+1),(i+1, j+1)$. Однако уравнениям второго порядка можно поставить в соответствие также цепочки, связывающие поведение последовательностей на большем числе точек решетки. Поэтому возникает вопрос о возможности обобщить метод на дискретные модели на большем количестве точек решетки,

\footnotetext{
1) $\mathrm{C}$ точки зрения приложений замыкания именно такого рода вызывают особый интерес.
} 
а также построить соответствующие примеры интегрируемых цепочек. Решению этой задачи и посвящена настоящая работа. Метод применим к задачам на четырех [5], пяти, семи (подобным рассматриваемым в работе [14]) и девяти точках решетки. Уравнения для моделей на семи и девяти точках решетки характерны значительной сложностью условий применимости метода и итеративных соотношений, определяющих преобразование Лапласа. Кроме того, соответствующий набор примеров интегрируемых нелинейных задач пока состоит лишь из систем разностных уравнений размерности не менее трех. Поэтому в настоящей работе целесообразно ограничиться рассмотрением задачи для моделей на пяти точках, а изучение задач на семи и девяти точках решетки рассмотреть в другой работе.

В разделе 2 вводятся понятия преобразования и инвариантов Лапласа для моделей на пяти точках. Раздел 3 посвящен обобщению результатов раздела 2 на системы дискретных уравнений: вводится понятие обобщенного инварианта Лапласа, исследуются условия его существования и корректность его определения. Показано, что именно обобщенные инварианты Лапласа обеспечивают адекватный критерий “лиувиллевости”. В разделе 4 приведен пример интегрируемой системы - обобщения двумеризованной цепочки Тоды и дискретного уравнения Лиувилля.

Как показано в работе [15], в отличие от скалярного случая, для систем нелинейных уравнений свойство конечности ряда инвариантов Лапласа (свойство “лиувиллевости") не эквивалентно интегрируемости по Дарбу, а является лишь ее необходимым условием. Тем не менее сравнительно несложно алгоритмизируемая процедура проверки “лиувиллевости" системы позволяет классифицировать системы типа Лиувилля как "вероятно интегрируемые по Дарбу", что с практической стороны оказывается весьма полезным для поиска интегралов.

\section{2. КАСКАДНЫЙ МЕТОД ДЛЯ СКАЛЯРНЫХ ЦЕПОЧЕК НА ПЯТИ ТОЧКАХ РЕШЕТКИ}

Рассмотрим линейное разностное уравнение с переменными коэффициентами на двумерной целочисленной решетке вида

$$
L_{0} u_{i j}=0, \quad L_{0}=T_{i} T_{j}+a_{i j} T_{j}+b_{i j} T_{i}+e_{i j} T_{-j}+g_{i j}=0, \quad i, j \in \mathbb{Z},
$$

$T_{i} u_{i j}=u_{i+1, j}, T_{j} u_{i j}=u_{i, j+1}, T_{-j} u_{i j}=u_{i, j-1}$. Перепишем оператор $L_{0}$ в следующем виде:

$$
\begin{aligned}
& L_{0}=\left(T_{i}+a_{i j}+c_{i j} T_{-j}\right)\left(T_{j}+b_{i-1, j}\right)+H_{i j}^{0}= \\
& =\left(T_{j}+b_{i j}\right)\left(T_{i}+a_{i, j-1}+c_{i j} \frac{b_{i-1, j-1}}{b_{i j}} T_{-j}\right)+K_{i j}^{0}, \\
& e_{i j}=c_{i j} b_{i-1, j-1}, \quad g_{i j}=a_{i j} b_{i-1, j}+c_{i j}+H_{i j}^{0},
\end{aligned}
$$


и по аналогии с классическим каскадным методом определим итеративную процедуру, задающую цепочки линейных уравнений:

$$
\begin{aligned}
L_{k}= & \left(T_{i}+a_{i, j+k}+c_{i j}^{k} T_{-j}\right)\left(T_{j}+b_{i-1, j}^{k}\right)+H_{i j}^{k}= \\
= & \left(T_{j}+b_{i j}^{k}\right)\left(T_{i}+a_{i, j+k-1}+c_{i j}^{k-1} T_{-j}\right)+K_{i j}^{k}, \\
b_{i j}^{0}= & b_{i j}, \quad c_{i j}^{-1}=c_{i j} \frac{b_{i-1, j-1}}{b_{i j}}, \quad k \in \mathbb{Z}, \\
& \left(T_{j}+b_{i j}^{k+1}\right) L_{k}=L_{k+1}\left(T_{j}+b_{i-1, j}^{k}\right),
\end{aligned}
$$

откуда определяем коэффициенты

$$
\begin{gathered}
b_{i j}^{k+1}=b_{i-1, j}^{k} \frac{H_{i, j+1}^{k}}{H_{i j}^{k}}, \quad c_{i j}^{k+1}=c_{i j}^{k} \frac{b_{i j}^{k+1}}{b_{i-1, j-1}^{k+1}}, \quad k \geqslant 0, \\
H_{i j}^{k+1}=H_{i, j+1}^{k}+a_{i, j+k} b_{i j}^{k+1}-a_{i, j+k+1} b_{i-1, j}^{k+1}+c_{i, j+1}^{k}-c_{i j}^{k+1}, \quad k \geqslant 0 .
\end{gathered}
$$

Аналогичным образом задается обратная процедура:

$$
\begin{gathered}
L_{k}=\left(T_{j}+b_{i+k, j}\right)\left(T_{i}+a_{i, j-1}^{k}+c_{i j}^{k} \frac{b_{i+k-1, j-1}}{b_{i+k, j}} T_{-j}\right)+K_{i j}^{k}, \\
a_{i j}^{0}=a_{i j}, \quad K_{i j}^{k-1}=H_{i-1, j}^{k}, \quad a_{i, j-1}^{k-1}=a_{i j}^{k} \frac{K_{i j}^{k-1}}{K_{i+1, j}^{k-1}}, \\
c_{i j}^{k-1}=c_{i j}^{k} \frac{K_{i, j-1}^{k-1}}{K_{i+1, j}^{k-1}} \frac{b_{i+k-1, j}}{b_{i+k-1, j-1}}, \quad k<0 .
\end{gathered}
$$

Нетрудно убедиться, что величины

$$
p_{i j}^{k}=\frac{H_{i j}^{k}}{a_{i, j+k} b_{i-1, j}^{k}}, \quad q_{i j}^{k}=\frac{H_{i j}^{k}}{c_{i j}^{k}}, \quad k \in \mathbb{Z},
$$

инвариантны относительно преобразования $u_{i j} \rightarrow \lambda_{i j} u_{i j}$, в дальнейшем будем называть их инвариантами Лапласа.

Основной интерес вызывает вопрос о применении каскадного метода к исследованию нелинейных цепочек, в связи с чем актуально следующее определение.

ОПРЕДЕЛЕНИЕ 2. Нелинейное дискретное уравнение

$$
u_{i+1, j+1}=f\left(u_{i j}, u_{i, j+1}, u_{i+1, j}, u_{i, j-1}\right)
$$

называется уравнением типа Лиувилля, если цепочки инвариантов Лапласа для его линеаризации

$$
\left(T_{i} T_{j}-\frac{\partial f}{\partial u_{i j}}-\frac{\partial f}{\partial u_{i+1, j}} T_{i}-\frac{\partial f}{\partial u_{i, j+1}} T_{j}-\frac{\partial f}{\partial u_{i, j-1}} T_{-j}\right) v_{i j}=0
$$

обрываются нулями с обеих сторон: $p_{i j}^{n}=p_{i j}^{m}=0$ для некоторых целых $n$ и $m$.

Нетривиальной задаче нахождения примеров нелинейных систем типа Лиувилля посвящен раздел 4. 


\section{3. СИСТЕМЫ ДИСКРЕТНЫХ УРАВНЕНИЙ НА ПЯТИ ТОЧКАХ РЕШЕТКИ}

Рассмотрим систему линейных разностных уравнений

$$
L_{0} u_{i j}=0, \quad L_{0}=T_{i} T_{j}+A_{i j} T_{j}+B_{i j} T_{i}+\widetilde{C}_{i j} T_{-j}+D_{i j}=0, \quad i, j \in \mathbb{Z},
$$

где $u_{i j}=\left(u_{i j}^{1}, u_{i j}^{2}, \ldots, u_{i j}^{n}\right)^{\mathrm{T}}$ - элемент неизвестных последовательностей векторов, $A_{i j}, B_{i j}, \widetilde{C}_{i j}, D_{i j}$ - некоторые $(n \times n)$-матрицы. Факторизуем оператор $L_{0}$ подобно тому, как это делалось в разделе 2 :

$$
L_{0}=\left(T_{i}+A_{i j}+C_{i j}^{0} T_{-j}\right)\left(T_{j}+B_{i-1, j}^{0}\right)+H_{i j}^{0},
$$

где $H_{i j}^{0}=D_{i j}-A_{i j} B_{i-1, j}^{0}-C_{i j}^{0}$. Итерации соответствующих преобразований Лапласа дают следующую процедуру:

$$
\begin{gathered}
L_{k}=\left(T_{i}+A_{i, j+k}+C_{i j}^{k} T_{-j}\right)\left(T_{j}+B_{i-1, j}^{k}\right)+H_{i j}^{k}= \\
=\left(T_{j}+B_{i j}^{k}\right)\left(T_{i}+A_{i, j+k-1}+C_{i j}^{k-1} T_{-j}\right)+K_{i j}^{k}, \\
\left(T_{j}+B_{i j}^{k+1}\right) L_{k}=L_{k+1}\left(T_{j}+B_{i-1, j}^{k}\right), \quad k=0,1,2, \ldots,
\end{gathered}
$$

откуда получаем уравнение для определения матриц $B^{k+1}, C^{k+1}, H^{k+1}$ :

$$
\begin{gathered}
B_{i j}^{k+1} H_{i j}^{k}=H_{i, j+1}^{k} B_{i-1, j}^{k}, \\
C_{i j}^{k+1} B_{i-1, j-1}^{k+1}=B_{i j}^{k+1} C_{i j}^{k}, \\
H_{i j}^{k+1}=H_{i, j+1}^{k}+B_{i j}^{k+1} A_{i, j+k}-A_{i, j+k+1} B_{i-1, j}^{k+1}+C_{i, j+1}^{k}-C_{i j}^{k+1} .
\end{gathered}
$$

Аналогичным образом может быть задана обратная процедура для $k<0$, что дает условия для определения величин $K_{i j}^{k}$.

Если матрица $H_{i j}^{k}$ невырожденна, то из уравнения (3.3) получаем матрицу $B_{i j}^{k+1}$. Если $B_{i j}^{k+1}$ тоже невырожденна, то из (3.4) получаем матрицу $H_{i j}^{k+1}$, что позволяет замкнуть цикл итераций. Однако в том случае, когда матрица $H_{i j}^{k}$ вырожденна и не совпадает тождественно с нулевой, встает вопрос о корректном определении $B_{i j}^{k+1}$. Во-первых, уравнение (3.3) может оказаться неразрешимым относительно $B_{i j}^{k+1}$, во-вторых, если оно разрешимо, то матрица $B_{i j}^{k+1}$ определяется неоднозначно. То же можно утверждать и по поводу уравнения (3.4). Перейдем теперь к обсуждению условий корректного определения цепочки инвариантов.

ОПРЕДЕЛЕНИЕ 3. Матрицу

$$
X_{i j}^{k}=H_{i+k, j}^{k} H_{i+k-1, j}^{k-1} \ldots H_{i j}^{0}, \quad k \geqslant 0,
$$

назовем обобщенным инвариантом системы (3.1).

Подобным образом определяется и второй обобщенный инвариант

$$
Y_{i j}^{k}=K_{i, j-k}^{-k} K_{i, j-k-1}^{-k-1} \ldots K_{i j}^{0}, \quad k<0 .
$$

Все рассуждения аналогичны для обоих инвариантов, поэтому приводим их только для величины $X_{i j}^{k}$. 
ЛЕмма 1. Пусть на $k$-м шаге процедуры определень матрицы $B_{i j}^{k} u C_{i j}^{k}$. Cuстемы уравнений

$$
\begin{aligned}
& B_{i+k, j}^{k+1} X_{i j}^{k}=X_{i, j+k}^{k} B_{i-1, j}^{0}, \\
& C_{i j}^{k+1} B_{i-1, j-1}^{k+1}=B_{i j}^{k+1} C_{i j}^{k}
\end{aligned}
$$

на матрицы $B_{i j}^{k+1}$ и $C_{i j}^{k+1}$ соответственно разрешимы тогда и толъко тогда, когда выполнены соответственно следующие условия:

$$
\begin{gathered}
B_{i-1, j}^{0} \operatorname{Ker} X_{i j}^{k} \subset \operatorname{Ker} X_{i, j+1}^{k}, \\
C_{i j}^{k} \operatorname{Ker} B_{i-1, j-1}^{k+1} \subset \operatorname{Ker} B_{i j}^{k+1} .
\end{gathered}
$$

Доказательство этой и следующих лемм основано на простых утверждениях линейной алгебры и аналогично доказательству, приведенному в работе [13] для цепочки на четырех точках решетки.

ЛЕмма 2. Пусть на $k$-м шаге процедуры выполнены следующие условия:

$$
\begin{gathered}
A_{i+l+1, j+l}^{\mathrm{T}} \operatorname{Ker}\left(X_{i+1, j}^{l}\right)^{\mathrm{T}} \subset \operatorname{Ker}\left(X_{i j}^{l}\right)^{\mathrm{T}}, \quad l=0,1, \ldots, k, \\
\operatorname{Ker}\left(X_{i j}^{0}\right)^{\mathrm{T}} \subset \operatorname{Ker}\left(X_{i-1, j-1}^{1}\right)^{\mathrm{T}} \subset \cdots \subset \operatorname{Ker}\left(X_{i-k, j-k}^{k}\right)^{\mathrm{T}}
\end{gathered}
$$

и для любого решения $B_{i j}^{k+1}$ системь (3.8)

$$
\operatorname{Ker}\left(B_{i+l, j-1}^{l+1}\right)^{\mathrm{T}} \subset \operatorname{Ker}\left(X_{i j}^{l}\right)^{\mathrm{T}}, \quad l=0,1, \ldots, k .
$$

Тогда обобщенный инвариант $X_{i j}^{k+1}$ не зависит от выбора матрич $B_{i j}^{l}, C_{i j}^{l}, l=$ $1,2, \ldots, k$.

Лемма 3. Пусть выполнены условия леммы 1 и леммы 2 и пусть на $k$-м шаге существуют такие матрицы $e_{i j}^{k}, d_{i j}^{k-1}, d_{i j}^{k}$, что $\left(e_{i j}^{k}\right)^{\mathrm{T}} \in \operatorname{Ker}\left(X_{i j}^{k-1}\right)^{\mathrm{T}},\left(d_{i j}^{l}\right)^{\mathrm{T}} \in$ $\operatorname{Ker}\left(B_{i-1, j-1}^{l}\right)^{\mathrm{T}}, l=k-1, k, u$

$$
H_{i+k, j}^{k}=e_{i j}^{k} A_{i+k, j+k-1}-A_{i+k, j+k} e_{i-1, j}^{k}+d_{i+k, j+1}^{k-1}-d_{i+k, j}^{k}+H_{i+k, j+1}^{k-1} .
$$

Тогда матрици $B_{i j}^{k}$ и $C_{i j}^{k}$ можно выбрать так, что соответствующая им матрица $H_{i j}^{k}$ равна нулю.

Отметим, что приведенные три леммы описывают условия возможности продолжения итеративной процедуры и оказываются нетривиальными лишь в случае вырождения матриц $H_{i j}^{k}$ и $B_{i j}^{k}$. Выполнение указанных условий необходимо проверять на каждом шаге итеративной процедуры.

\section{4. ДИСКРЕТНОЕ УРАВНЕНИЕ ЛИУВИЛЛЯ НА ПЯТИ ТОЧКАХ РЕШЕТКИ}

Нахождение нетривиальных примеров нелинейных цепочек типа Лиувилля является непростой задачей. Воспользуемся закономерностью, которую можно отметить при изучении непрерывных моделей вида (1.1), (1.6) и дискретных цепочек 
на четырех точках решетки вида (1.8) и (1.12). Во всех случаях нетривиальные уравнения со свойством “лиувиллевости" (конечность ряда инвариантов Лапласа) получаются путем наложения простых краевых условий на итеративные соотношения, связывающие инварианты Лапласа. Например, соотношение (1.5) при условии $0=h_{ \pm 1}=h_{ \pm 2}=\cdots$ дает само уравнение Лиувилля на величину $h_{0}$, дискретный аналог уравнения Лиувилля (1.10) получается из соотношения (1.9) с такими же краевыми условиями. Аналогичные результаты характерны и для систем уравнений (см. [13], [16]). Поэтому естественно выполнить подобные операции и для нашего случая моделей на пяти точках решетки.

Запишем итерационное соотношение, связывающее члены двух последовательностей инвариантов Лапласа для уравнения (2.1):

$$
p_{i j}^{k}=\frac{H_{i j}^{k}}{a_{i, j+k} b_{i-1, j}^{k}}, \quad q_{i j}^{k}=\frac{H_{i j}^{k}}{c_{i j}^{k}}, \quad k \in \mathbb{Z},
$$

при условии выполнения связей (2.5). Таким образом, получаем следующую системy:

$$
\begin{aligned}
p_{i j}^{k-1}+1 & =\frac{1}{r_{i j}^{k}}\left(1+\frac{1}{p_{i j}^{k}}+\frac{1}{q_{i j}^{k}}-\frac{r_{i, j+1}^{k}}{p_{i j}^{k} q_{i, j+1}^{k}}\right), \\
p_{i j}^{k+1}+1 & =\frac{p_{i-1, j}^{k} p_{i, j+1}^{k}}{r_{i-1, j+1}^{k}}\left(1+\frac{1}{p_{i j}^{k}}+\frac{1}{q_{i, j+1}^{k}}-\frac{p_{i-1, j-1}^{k}}{r_{i-1, j}^{k} q_{i j}^{k}}\right), \\
q_{i j}^{k-1}+1 & =\frac{p_{i, j-1}^{k} q_{i j}^{k}}{r_{i j}^{k}}\left(1+\frac{1}{p_{i, j-1}^{k}}+\frac{1}{q_{i, j-1}^{k}}-\frac{1}{r_{i, j-1}^{k}}\right), \\
q_{i j}^{k+1}+1 & =\frac{r_{i-1, j}^{k} q_{i j}^{k}}{p_{i-1, j-1}^{k}}\left(1+\frac{1}{p_{i j}^{k}}+\frac{1}{q_{i, j+1}^{k}}-\frac{r_{i-1, j+1}^{k}}{p_{i-1, j}^{k} p_{i, j+1}^{k}}\right),
\end{aligned}
$$

где

$$
r_{i j}^{k}=\frac{H_{i j}^{k}}{a_{i, j+k-1} b_{i j}^{k}} .
$$

Запишем эту систему в виде набора уравнений:

$$
\begin{gathered}
p_{i j}^{k+1}=\alpha_{i j} \frac{p_{i, j+1}^{k} p_{i-1, j}^{k}}{r_{i-1, j+1}^{k}}, \quad q_{i j}^{k+1}=\alpha_{i j} \frac{q_{i j}^{k} r_{i-1, j}^{k}}{p_{i-1, j-1}^{k}}, \quad r_{i j}^{k+1}=\alpha_{i j} p_{i j}^{k}, \\
p_{i j}^{k-1}=\beta_{i j}, \quad q_{i j}^{k-1}=\beta_{i, j-1} \frac{q_{i j}^{k} p_{i, j-1}^{k}}{r_{i, j-1}^{k} r_{i j}^{k}}, \quad r_{i j}^{k-1}=\frac{\beta_{i, j-1} \beta_{i+1, j}}{\beta_{i+1, j-1}} \frac{r_{i+1, j-1}^{k}}{p_{i+1, j-1}^{k}},
\end{gathered}
$$

где

$$
\begin{aligned}
& \alpha_{i j}=1+\frac{1}{p_{i j}^{k}}+\frac{1}{q_{i, j+1}^{k}}-\frac{r_{i-1, j+1}^{k}}{p_{i-1, j}^{k} p_{i, j+1}^{k}}-\frac{p_{i-1, j-1}^{k}}{q_{i j}^{k} r_{i-1, j}^{k}} \\
& \beta_{i j}=r_{i j}^{k}\left(1+\frac{1}{p_{i j}^{k}}+\frac{1}{q_{i j}^{k}}-\frac{r_{i, j+1}^{k}}{q_{i, j+1}^{k} p_{i j}^{k}}\right)-1 .
\end{aligned}
$$


Из (4.3), исключая зависимую величину $r_{i j}^{k}$, выводим следующую систему из двух нелинейных уравнений на две неизвестные последовательности:

$$
\begin{gathered}
\left(p_{i, j+1}^{k-1}+1+\frac{p_{i, j+1}^{k-1}}{q_{i, j+2}^{k-1}}\right)\left(p_{i+1, j}^{k+1}+1+\frac{p_{i+1, j}^{k+1}}{q_{i+1, j}^{k+1}}\right)= \\
=p_{i j}^{k} p_{i+1, j+1}^{k}\left(1+\frac{1}{p_{i+1, j}^{k}}+\frac{1}{q_{i+1, j+1}^{k}}\right)\left(1+\frac{1}{p_{i, j+1}^{k}}+\frac{1}{q_{i, j+1}^{k}}\right), \\
\frac{p_{i j}^{k-1} p_{i+1, j}^{k+1}}{q_{i, j+1}^{k-1} q_{i+1, j}^{k+1}}=\frac{p_{i, j-1}^{k} p_{i+1, j+1}^{k}}{q_{i+1, j}^{k} q_{i, j+1}^{k}},
\end{gathered}
$$

которую по аналогии с работой [5] уместно назвать дискретным векторным аналогом двумеризованной цепочки Тоды. Отметим также, что условие $c_{i j}^{k}=0(1 / q=0)$ сводит эту систему к двумеризованной цепочке Тоды (1.9), полученной в работе [5]. Естественно ожидать, что, как и в непрерывном (1.5) и дискретном (1.9) скалярных случаях, наложения простых краевых условий (замыканий) на решения двумеризованной цепочки Тоды приведут к ряду нетривиальных нелинейных цепочек типа Лиувилля на пяти точках решетки.

Пример. Определим простейшее замыкание для системы (4.4):

$$
p_{i j}^{k}=p, \quad q_{i j}^{k}=q, \quad k \neq 0,
$$

$p, q$ - ненулевые константы. Тогда имеем систему на две последовательности $p_{i j}=$ $p_{i j}^{0}, q_{i j}=q_{i j}^{0}$ :

$$
\begin{gathered}
p_{i j} p_{i+1, j+1}\left(1+\frac{1}{p_{i+1, j}}+\frac{1}{q_{i+1, j+1}}\right)\left(1+\frac{1}{p_{i, j+1}}+\frac{1}{q_{i, j+1}}\right)=p^{2}\left(1+\frac{1}{p}+\frac{1}{q}\right)^{2}, \\
\frac{p_{i, j-1} p_{i+1, j+1}}{q_{i+1, j} q_{i, j+1}}=\left(\frac{p}{q}\right)^{2}
\end{gathered}
$$

которая легко приводится к системе на пяти точках решетки вида (2.8). При наложении дополнительного условия $1 / q_{i j}=1 / q=0$ система (4.5) сводится к дискретному уравнению Лиувилля (1.10) и в предельном переходе превращается в само уравнение Лиувилля. Поэтому естественно назвать систему (4.5) дискретным аналогом уравнения Лиувилля на пяти точках решетки. При этом “лиувиллевость" системы (4.5) понимается в смысле положений раздела 3. С помощью символьных вычислений, использующих описанную схему линеаризации и вычисления инвариантов, можно показать, что обобщенный инвариант Лапласа для системы (4.5) равен нулю. Как уже упоминалось, в работе [15] показано, что в векторном случае, в отличие от скалярного, свойство “лиувиллевости" нелинейного уравнения не эквивалентно свойству интегрируемости по Дарбу, а является лишь ее необходимым условием. По аналогии с этой работой сформулируем следующее утверждение.

УтВЕРЖДЕНИЕ. Пусть у системы (4.5) имеется набор интегралов, т.е. двумерный вектор

$$
w\left(j, u_{i j}, u_{i, j \pm 1}, u_{i, j \pm 2}, \ldots, u_{i, j \pm m}\right), \quad u_{i j}=\left(p_{i j}, q_{i j}\right)^{\mathrm{T}},
$$


такой, что $T_{i} w=0$ и матрища $\partial w / \partial u_{i, j \pm m}$ невырожденна $и$ для всех $j \leqslant m$ существуют и однозначно определены инварианты Лапласа $X_{j}$. Тогда $X_{m}=0$.

Таким образом, дискретный аналог уравнения Лиувилля (4.5) является системой типа Лиувилля, и, следовательно, необходимое условие интегрируемости по Дарбу выполняется, т.е. существует набор интегралов по характеристическим направлениям.

Благодарности. Работа выполнена при частичной поддержке РФФИ (гранты № 07-01-00081, 05-01-97910-р-агидель-а) и Программы Президиума РАН “Фундаментальные проблемы нелинейной динамики".

\section{Список литературы}

[1] G. Darboux, Leçons sur la théorie des surfaces, II, Gauthier-Villars, Paris, 1889.

[2] I. M. Anderson, N. Kamran, Duke Math. J., 87:2 (1997), 265-319.

[3] А. В. ЖКибер, В. В. Соколов, С. Я. Старцев, Докл. РАН, 343:6 (1995), 746-748.

[4] А. В. Жибер, В. В. Соколов, Метод каскадного интегрирования Лапласа и уравнения, интегрируемые по Дарбу, РИЦ БашГУ, Уфа, 1996.

[5] В.Э. Адлер, С. Я. Старцев, ТМФ, 121:2 (1999), 271-284.

[6] А. В. Жибер, В. В. Соколов, УМН, 56:1(337) (2001), 63-106.

[7] А. М. Гурьева, Метод каскадного интегрирования Лапласа и нелинейные гиперболические системы уравнений, Дис. ... канд. физ.-мат. наук, УГАТУ, Уфа, 2005.

[8] R. Hirota, J. Phys. Soc. Japan, 46:1 (1979), 312-319.

[9] R. Hirota, J. Phys. Soc. Japan, 50:11 (1981), 3785-3791.

[10] D. Levi, L. Pilloni, P. M. Santini, J. Phys. A, 14:7 (1981), 1567-1575.

[11] R. S. Ward, Phys. Lett. A, 199:1-2 (1995), 45-48.

[12] A. Doliwa, Phys. Lett. A, 234:3 (1997), 187-192.

[13] В. Л. Верещагин, ТМФ, 156:2 (2008), 207-219.

[14] С. П. Новиков, И. А. Дынников, УМН, 52:5(317) (1997), 175-234.

[15] А.В. ЖКибер, В.В. Соколов, С.Я. Старцев, "Нелинейные гиперболические системы лиувиллевского типа", Материаль международной конференции “Тихонов-100”, Т. 1, $2006,1-2$.

[16] А. М. Гурьева, А. В. Жибер, ТМФ, 138:3 (2004), 401-421.

Поступила в редакцию 13.08.2008, после доработки 5.11.2008 